(e-migrinter

\section{e-Migrinter}

$13 \mid 2015$

Mobilités, circulation migratoire et transnationalisme au prisme des réflexions méthodologiques de jeunes chercheur(e)s en sciences sociales

\title{
Du contexte villageois au village global
}

approche réflexive de la contribution théorique d'une recherche empirique sur la migration internationale (Cadaqués, Espagne)

Josepha Milazzo

\section{(Q) OpenEdition \\ Journals}

Édition électronique

URL : https://journals.openedition.org/e-migrinter/545

DOI : 10.4000/e-migrinter.545

ISSN : 1961-9685

Éditeur

UMR 7301 - Migrinter

Référence électronique

Josepha Milazzo, «Du contexte villageois au village global », e-Migrinter [En ligne], 13 | 2015, mis en

ligne le 06 octobre 2015, consulté le 20 mai 2021. URL : http://journals.openedition.org/e-migrinter/ 545 ; DOl : https://doi.org/10.4000/e-migrinter.545

Ce document a été généré automatiquement le 20 mai 2021.

Tous droits réservés 


\section{Du contexte villageois au village global}

approche réflexive de la contribution théorique d'une recherche empirique sur la migration internationale (Cadaqués, Espagne)

Josepha Milazzo

\section{NOTE DE L'AUTEUR}

J'aimerais adresser mes sincères remerciements aux habitants de la localité de Cadaqués pour avoir partagé leurs expériences, sans leur contribution, mon travail de thèse n'aurait simplement pas pu voir le jour. Je remercie vivement le Social Science Research Council et le laboratoire Migrinter de l'Université de Poitiers pour m'avoir donnée l'opportunité heureuse depuis deux ans de participer à des rencontres scientifiques dans le domaine des études migratoires, qui ont jouées un rôle déterminant dans l'évolution de mon travail de recherche, notamment dans le cadre du programme DPDF « New Approaches to Transnationalism and Migratory Circulation » (2012) et «Alumni in International Fields in Migration Studies » (2014), et de plusieurs séminaires organisés conjointement, dont celui à l'origine de ce numéro. À ce titre, je souhaiterais adresser ma gratitude à Peggy Levitt pour ses remarques constructives et à Thomas Lacroix pour son implication. J'exprime également ma reconnaissance à mes directeurs de thèse, Virginie Baby-Collin et Ricard Morén-Alegret, pour leurs conseils éclairés et leur soutien. Pour finir, j'adresse un grand merci aux autres chercheurs-auteurs qui ont participé à l'élaboration de ce numéro - une expérience enrichissante - aux relecteurs anonymes ainsi qu'à Anne Bouhali.

1 L'Espagne est devenue depuis une quinzaine d'années une destination importante pour la migration internationale de travail des Boliviens. Toutefois l'Argentine demeure le pays d'accueil traditionnel des Boliviens (Cortes, 2000), il en est de même pour les États-Unis d'Amérique. Dans le paysage migratoire espagnol, qui connaît un phénomène de latino-américanisation, la présence bolivienne n'est pas, par ailleurs, 
numériquement prédominante. Cette migration se caractérise surtout par la soudaineté de son apparition, et par la rapidité de la réorientation géographique et de l'évolution numérique des flux, consécutivement à divers événements politiques, économiques et géopolitiques à travers le monde (Baby-Collin, Cortes, Miret, 2009). À l'échelle de l'Espagne, les migrants boliviens se concentrent dans les pôles urbains madrilène et barcelonais. Néanmoins, certaines petites localités se voient également concernées par cette présence bolivienne. Dans des espaces excentrés de ces localités la part des Boliviens y est la plus importante. La présence bolivienne dans ces municipios périphériques et moins peuplées, pose alors la question de l'attractivité de ces derniers et de ce qui s'y passe. Quelles y sont les pratiques et les constructions socio-spatiales, liées à l'immigration? Y sont-elles spécifiques, différentes de celles observables dans les grands pôles urbains espagnols?

2 J'expose d'abord comment le dialogue entre empirie et théorie a re-circonscrit mon sujet et mes objectifs de recherche, me conduisant à formuler de nouveaux questionnements. Par la suite, j'ai ainsi déterminé ma problématique à partir d'une approche inductive appliquée à mon terrain. J'ai finalement proposé d'apporter une contribution théorique en clarifiant le concept de «village global» selon l'une des réappropriations dont il avait fait l'objet.

\section{D'une lecture transnationale des migrations internationales à l'approche paradigmatique d'un village comme lieu d'immigration}

3 Je discute ci-après quels sont les enjeux de ma thèse, replacés dans une présentation succincte de la littérature associée. Mes objectifs de recherche ont évolué au gré d'un changement du paradigme de lecture de la migration internationale.

\section{Contextualisation : sujet et objectifs de recherche actuels}

Dans un cadre conceptuel issu de la géographie sociale et de la sociologie de la mondialisation, avec l'appui de méthodologies propres à la géographie culturelle, à l'anthropologie et à la sociologie urbaine et dans la lignée thématique d'une géographie des relations (relations urbain-rural, circulations, réseaux, pratiques), l'un des objectifs de ma thèse en géographie est d'apporter des réserves quant aux paradigmes urbains dominants en m'intéressant aux perspectives propres des études migratoires à l'échelle de petites localités excentrées et peu peuplées, dans une dynamique Sud-Nord. J'interroge le rôle de la migration internationale dans les transformations et le repositionnement potentiel de telles communes au sein d'une hiérarchie mondiale concurrentielle dominée par les villes.

Ma thèse s'appuie sur l'étude des modes d'habiter et de travailler de migrants boliviens, dans la petite municipalité espagnole de Cadaqués. Celle-ci est située dans la région la plus orientale de la péninsule ibérique, à l'extrémité nord-est de la Costa Brava. Elle dispose d'un accès direct à la mer Méditerranée et est relativement isolée du reste de l'arrière-pays par des massifs montagneux. Cette configuration spatiale explique que la commune soit restée pratiquement isolée du reste du continent jusqu'à la fin du $19^{\text {ème }}$ siècle, et qu'elle continue d'être difficilement accessible en raison d'une seule voie de 
communication terrestre. Avec un noyau villageois historique, le reste du bâti de la commune est relativement résiduel et éparse, si ce n'est quelques développements résidentiels et touristiques récents et un ancien port de pêche, Port Lligat. Ce noyau villageois s'est maintenu, densifié et s'est très peu étalé, compte tenu de la superficie totale de l'espace communal, de ses contraintes physiques et d'un développement urbain maîtrisé, ceci dès la mise en valeur touristique généralisée de la Costa Brava amorcée dans les années 1950, et a fortiori avec la mise en place de l'espace protégé du parc naturel terrestre et maritime du Cap de Creus en 1998. Cadaqués a ainsi connu une reconversion de sa mise en valeur économique, l'agriculture et la pêche, anciennement dominantes, laissant place au secteur touristique. Il serait ainsi difficile de parler d'économie rurale à proprement parler dans le contexte actuel. Néanmoins, il ne s'agit pas ici d'ouvrir un débat sur la ruralité ou l'urbanité de Cadaqués, qui est une question que j'aborde par ailleurs dans le cadre de ma thèse. Je préciserai simplement, pour contextualiser l'argumentaire, que Cadaqués peut être défini à plus d'un titre comme un "village ", en raison du bâti dense et limité à l'expansion urbaine de son noyau originel qui concentre une majorité des habitants locaux, dont l'effectif est inférieur à 5000 habitants résidents à l'année ${ }^{1}$. C'est à ce titre que l'Institut National de Statistiques espagnol (I.N.E.) définit, à l'échelle nationale, la municipalité de Cadaqués comme village (pueblo), et originellement comme vila ${ }^{2}$, selon une acception davantage politique.

Quelles sont alors les modalités de transformations du village de Cadaqués dans un contexte de mondialisation? L'insertion de nouveaux acteurs au sein de ce territoire, notamment avec l'immigration de communautés étrangères en général et boliviennes en particulier, interroge sur leur possible influence dans ces dynamiques. Je cherche ainsi à montrer comment la migration internationale participe à la reconstruction de la localité à Cadaqués, en interrogeant le tourisme comme vecteur principal de la mise en valeur différenciée de ce territoire.

7 Mon argumentaire repose sur la documentation statistique et bibliographique, l'analyse de soixante entretiens semi-directifs et d'observations participantes réalisés durant les étés 2012 et 2013 auprès de différentes communautés étrangères et de la population locale, résidentes de Cadaqués, dont une majorité de migrants boliviens. J’ai aussi réalisé un travail ethnographique en vivant auprès de deux familles boliviennes durant ces deux étés. Par ailleurs, durant le printemps 2011, j'ai également mené des enquêtes auprès de familles de migrants, de retour d'Espagne ou ayant des membres en Espagne, dans la ville bolivienne de Cochabamba.

8 Ma thèse ayant connu une reformulation majeure, il est nécessaire de revenir sur ces changements pour comprendre dans quelle mesure sujet et objectifs de recherche se reconstruisent au gré de facteurs divers, selon, en autres, les moyens financiers et temporels disponibles pour la réalisation d'une thèse dans le cadre de "plusieurs terrains dans le terrain", mais également selon les intérêts intellectuels, ou encore selon l'orientation donnée à la recherche par les directeurs.

\section{Rétrospective d'un recadrage des intérêts de recherche}

9 D'une approche transnationale des pratiques des migrants boliviens entre la Bolivie et l'Espagne, avec deux terrains, l'un à Cochabamba, dans l'espace d'origine, et l'autre à Cadaqués, dans l'espace de réception, mon intérêt s'est recentré sur les dynamiques 
migratoires en contexte autre qu'urbain et sur le rôle de la migration internationale dans les transformations que peuvent ainsi connaître ces autres types de localités ${ }^{4}$. Cadaqués est ainsi devenu l'espace référent de mes recherches et le lieu d'observation d'une part de la participation des migrants à ces évolutions éventuelles, et d'autre part, des relations s'y déroulant.

Cette réorientation s'explique par un constat : nombre de travaux s'accordent sur le fait que certaines grandes villes et villes globales (Sassen, 2001) demeurent jusqu'à présent les points nodaux d'une économie d'archipel (Veltz, 1996) et les principaux sites d'observation des interactions entre la mondialisation et la migration (Benton-Short, Price, Friedman, 2005), vues depuis les espaces d'arrivée. Toutefois, des travaux ont commencé à s'intéresser à la migration internationale à destination d'autres types de localités : ils soulignent la nécessité de rechercher la spécificité des lieux, des espaces et des pratiques en jeu et dont la théorisation doit précisément être faite en lien avec la migration (Glick Schiller et Caglar, 2011, 63). Dans le contexte actuel de mondialisation migratoire ${ }^{5}$ (Simon, 2008), ces travaux appellent à la considération du local pour une compréhension des modalités d'ancrage spatial de processus se déroulant à l'échelle mondiale. Ils renvoient à la question de la différenciation spatiale au cœur du débat actuel sur la mondialisation qui est entendue en géographie comme «la maîtrise de l'espace par les différents acteurs (États, sociétés, entreprises) et [comme] la valorisation différenciée et sélective des différents territoires par le capital dans un cadre concurrentiel » (Carroué, 2006, 7). Sur ce point, je partage l'argument de Becky Mansfield $(2003,3)$, selon lequel « spatialiser la mondialisation ce n'est pas seulement poser en principe la spécificité locale, mais c'est aussi prêter attention à la façon dont les pratiques économiques [entre autres] sont spatialement différenciées ${ }^{6}$ : la mondialisation ne se réalise pas de manière identique à l'échelle planétaire, et l'augmentation des échanges contribuerait à produire des différences, plutôt qu'une homogénéisation des lieux.

11 Aussi, ce qui demeure encore peu étudié dans les études migratoires, ce sont les contextes villageois et les petites localités métropolitaines et non-métropolitaines de l'immigration internationale, comme autant d'espaces d'arrivée et de transit pour les migrants internationaux et les immigrés étrangers, bien que des travaux dans la même veine commencent également à se développer, à la recherche d'éléments qui soient spécifiques au milieu d'immigration (voir Morén-Alegret, Solana, 2004 ; Fonseca, 2008 ; Hugo, 2008 ; Hugo, Morén-Alegret, 2008 ; Kasimis, 2008 ; Morén-Alegret, 2008 ; Jentsch, Simard, 2009; Marrow, 2011; Nadler, 2012 ; Bayona-i-Carrasco, Gil-Alonso, 2013). D'autres études s'intéressent plus spécifiquement aux processus de mondialisation rurale (Woods, 2007 ; McCarthy, 2008) en lien avec la migratoire internationale (Hogan, 2004), comme par exemple l'étude de la circulation internationale à destination d'espaces ruraux qui constituent autant de niches économiques pour des migrants internationaux qui y travaillent généralement dans l'agriculture intensive.

Comment interroger cette spécificité supposée des phénomènes liés au milieu d'immigration à Cadaqués et la justifier sans cadre comparatif autre que celui de la littérature traitant d'autres localités? Le sujet de ma recherche, s'intéresser aux dynamiques migratoires dans une petite localité excentrée et peu peuplée, était clair. Cependant, la détermination de mes objectifs de recherche m'échappait. Je n'avais pas de problématique définie. Je prenais ainsi le parti du terrain selon une approche 
inductive pour m'apporter une clef de lecture de la particularité éventuelle des modalités d'inscription des dynamiques migratoires à Cadaqués.

\section{Migrations internationales, contexte villageois et localité : ma problématique déterminée à l'épreuve d'une approche inductive du terrain de recherche}

13 J'expose ci-après quelles ont été les modalités de mon entrée sur le terrain à Cadaqués. L'objectif était alors, au gré des phénomènes observés, de déterminer une problématique venant éclairer mon sujet de recherche, à savoir, la spécificité de la présence des migrants boliviens à Cadaqués.

\section{À la recherche d'une spécificité du milieu d'immigration}

J'ai contourné les difficultés liées à la recherche d'une spécificité liée au milieu d'immigration en choisissant comme angle d'attaque de m'intéresser aux effets de lieu ${ }^{7}$ (Doceul, Tabarly, 2014) pour interroger le contexte villageois de Cadaqués comme lieu d'inscription de dynamiques migratoires internationales. La démarche consiste à dépasser la question de contextualisation du «où? " et du "quand? " pour plutôt poser celles du «pourquoi ici ? " et du " avec quels effets?", ouvrant ainsi la porte à l'éventuelle mise en évidence d'une spécificité des processus étudiés. Me plaçant dans une démarche inductive, je ne souhaitais pas préparer outre mesure ce terrain pour rester « en tant que chercheur actif, passif vis-à-vis de ses théories d'arrière-plan au sens où il cherche à ne pas être déterminé par elles et à rester ouvert à la découverte " (Dumez, 2012, 8). Mon entrée en terrain allait donc être cadrée par ces deux questionnements principaux : pourquoi une immigration bolivienne à Cadaqués ? Quels sont les effets de cette immigration? En l'occurrence, le contexte de Cadaqués est triplement particulier car il s'agit d'un village, il présente une configuration rappelant le milieu insulaire, et pour finir on pourrait le qualifier de village global (comme je l'expliciterai par la suite). Je pouvais en effet voir, au gré de mon terrain, dans quelle mesure Cadaqués s'écartait d'une définition classique du village, entendu comme "petit, moins urbanisé, peu peuplé, avec peu de services, avec de petites industries souvent liées à l'agriculture et aux professions de l'artisanat, davantage lié à la culture locale traditionnelle, plus homogène, plus personnel, avec moins de poids politique et économique, plus équilibré avec son arrière-pays rural, etc. $»^{8}$ (Afshar, 1998, 376). Trois points peuvent être à ce titre soulignés, pour éclairer les enjeux majeurs de mon sujet de recherche et donner des éléments complémentaires de contextualisation.

D'abord, la configuration géographique spécifique de Cadaqués, commune située sur une presqu'île, explique que, pareillement à une grande partie de l'Espagne, le village ait d'abord été un lieu d'émigration, notamment à destination des Amériques, et dont on peut encore voir aujourd'hui une trace dans l'architecture de certaines bâtisses construites par des migrants de retour de Cuba. Mais l'évolution de ce village s'inscrit dans une histoire migratoire beaucoup plus longue avec, depuis une vingtaine d'années, l'immigration latino-américaine qui vient complexifier le paysage migratoire espagnol et qui n'a pas épargné Cadaqués où les Boliviens, première nationalité 
étrangère locale ${ }^{9}$, arrivent pareillement, par le biais de filières migratoires. Pour rappel, à l'échelle de l'Espagne, ils constituent l'un des récents groupes immigrés.

Ceci nous amène à un second point : qui sont ces migrants et que viennent-ils faire à Cadaqués? Cadaqués est un village très touristique et dont l'économie locale repose essentiellement sur ce secteur. L'isolement géographique de Cadaqués a travaillé en faveur d'une préservation de son environnement, du maintien de son architecture et du développement d'un tourisme culturel haut de gamme, dans une certaine mesure comme l'héritage du peintre Salvador Dali qui y a vécu une partie de sa vie, un tourisme sur lequel repose la globalité, l'attractivité et le rayonnement de Cadaqués. Les flux touristiques à Cadaqués sont importants durant la période estivale ${ }^{10}$ et nécessitent le recrutement de nombreux employés dans la restauration, l'hôtellerie, la construction, etc., parmi lesquels figurent des migrants internationaux qui se trouvent être les travailleurs locaux de la mondialisation.

Cadaqués présente ainsi différents mondes sociaux qui coexistent dans cet espace ayant conservé une identité spécifique catalane et locale très forte, portée aussi bien par ses habitants que par le pouvoir municipal, et qui s'explique en partie par le caractère insulaire de la commune. Ceci conduit à une tension entre, d'un côté, l'affirmation d'une spécificité identitaire locale, renforcée parce qu'elle est notamment promue par la politique touristique locale, et de l'autre, l'existence d'une multitude d'apports cosmopolites. Les touristes, les artistes internationaux et les migrants de diverses nationalités dont des Boliviens, des Équatoriens, des Marocains et une vingtaine d'autres nationalités présentes, travaillent tous dans cette économie. Bien sûr, une partie de ces acteurs est contrainte à une certaine invisibilité et arrive difficilement à se faire une place.

Cette mise en valeur économique et cette diversité étrangère à l'échelle d'un espace géographiquement enclavé, avec une forte densité de 1000 habitants au $\mathrm{km}^{2}$, ont progressivement façonné l'idée $\mathrm{du}$ "monde » condensé dans un village qui pouvait alors être qualifié de "global». Cadaqués présente en effet une forte coprésence de communautés étrangères immigrées et pose ainsi la question des pratiques relationnelles qui s'y déploient et de leurs implications sur l'organisation territoriale. M'interroger sur les raisons de l'immigration bolivienne à Cadaqués et sur la nature des effets associés m'amena ainsi à reconsidérer le rôle des migrants dans l'évolution du village et dans les transformations connues par ce dernier sur le temps long, depuis la mise en valeur touristique de la Costa Brava dans les années 1950-1960, et plus particulièrement à partir des années 2000, époque d'arrivée des premiers Boliviens à Cadaqués coïncidant avec un tournant vers un tourisme de plus en plus important au sein du village. Je pouvais en effet constater, à l'aune du terrain effectué, l'importance de la présence de processus mondiaux s'inscrivant dans cette petite localité. Ceci m'amena à m'interroger sur les enjeux que cette configuration pouvait soulever.

\section{La (re)production de la localité en lien avec la mondialisation et le rôle de la migration internationale dans celle-ci}

19 Sur ces bases et après un retour à la littérature scientifique, je creusais le rôle de la migration internationale, parmi d'autres processus mondiaux, dans les transformations socio-spatiales que pouvait connaître le village de Cadaqués. La migration, en tant que l'une des formes de la mondialisation, doit être analysée comme un élément central des 
transformations sociales globales ${ }^{11}$ (Castles, 2000). Les objectifs de ma thèse, tels que fixés, étaient alors de décrypter le fonctionnement du système propre à Cadaqués (que je qualifierai par la suite de village global). Dans ce but, je me suis plus particulièrement intéressée aux vies des migrants (notamment boliviens) et à leur rôle dans les fonctionnements communautaires et les relations humaines à Cadaqués, comme suggéré par ma problématique : «dans un contexte caractérisé par les mondialisations touristique, culturelle, économique et migratoire et un nationalisme régional et local important, comment la présence éclectique de migrants internationaux intervient-elle dans la renégociation du modèle de production de la localité à l'œuvre dans le village global de Cadaqués?»

Je me place dans la continuité des travaux d'Arjun Appadurai $(2005,274-275)$ sur « la modalité relationnelle de la production globale et située de la localité $»^{12}$ qui adapte des éléments qui lui sont de prime abord exogènes. Selon Appadurai (2005), cette production peut être relativement complexifiée par «le mouvement humain», qu'il soit « lié au travail qui pousse les gens à émigrer » ou " aux industries du loisir ${ }^{13}$ qui en attirent d'autres, ce qui aboutit à un «brassage d'éléments exogènes au sein d'un même lieu ». Ce point est relativement pertinent dans le cas de Cadaqués, dont la reproduction de la localité est précisément sujette à un fort brassage d'éléments exogènes dans le cadre d'une mise en valeur touristique de l'espace, attirant des touristes et des travailleurs migrants étrangers. Nina Glick-Schiller (2011) qui appelle à une analyse située de la participation de la migration à la reproduction de la localité, évoque deux points importants quant aux processus de (re)production de la localité et au rôle que peut y jouer la migration. D'une part, elle souligne la relation réciproque, rétroactive, de reconfiguration mutuelle entre les dynamiques migratoires et certaines transformations de la localité. D'autre part, elle évoque l'importance explicative « des couches complexes de l'histoire sociale et de la structure sociale [d'un contexte dans] les processus en lien avec la variation [observable d'un cas d'étude à un autre] de l'incorporation [située] des migrants $»^{14}$ (Glick Schiller, Çağlar, 2011, 80) et qui peuvent ainsi conduire à des impacts différenciés de la migration dans la restructuration du tissu social selon les cas étudiés.

D'un point de vue méthodologique, on comprend d'autant mieux comment, à partir de ces considérations, un objet géographique résulte d'un processus de construction que l'on observe dans le temps, avant qu'il ne puisse constituer un objet transposable à d'autres espaces. En cela, il témoigne dans une certaine mesure de l'état d'une culture à un moment donné. Il peut devenir un construit cognitif qui permet d'appréhender un phénomène spatial dès lors que l'on interroge sa pertinence dans un contexte donné, en fonction d'une problématique géographique. Mon objectif était alors de considérer le concept de village global en tant qu'objet géographique d'analyse pour interroger sa pertinence dans le contexte donné de Cadaqués.

\section{Le village global, prisme conceptuel d'analyse de la participation des migrants internationaux aux transformations locales d'un village de réception}

$\mathrm{Au}$ gré de mes observations, et dans le but d'apporter, par mes travaux, une contribution théorique, j'ai ainsi pensé clarifier le concept de village global en l'élevant 
au rang de géotype ${ }^{15}$, pour en faire un objet générique. Comment le village global a-t-il été originellement conceptualisé et diversement réapproprié dans les travaux empiriques?

\section{Du global village, monde connecté à l'échelle planétaire, au « village global », petite localité influente dans la mondialisation}

Le concept de village global a originellement été développé par le spécialiste canadien des médias et de la communication Marshall McLuhan (1962) pour métaphoriser les effets de la technologie communicationnelle sur une psychologie humaine désireuse d'être connectée à l'échelle planétaire en un seul et même "village ». Selon lui, l'avènement de l'électricité et de la télévision, l'ère des médias, bien avant celle de la mondialisation et des nouvelles technologies de l'information et de la communication (NTIC), inciterait à l'avenir les gens à développer un type de sensibilité et de participation comparables à celles des petites communautés, renouant ainsi avec les idées de proximité physique et psychologique, de ressemblance, propres aux sociétés villageoises. Mon travail n'entend pas joindre une conversation sur les liens entre communication et mondialisation. Cependant, cette question des enjeux relationnels en contexte villageois constitue une piste de réflexion féconde à creuser, dès lors que l'on met en parallèle deux acceptions et métaphores possibles du village global : d'un côté, l'espace mondial est connecté et perçu comme un unique et même "village", selon McLuhan (1962). De l'autre, comme le suggère le cas d'étude de Cadaqués, le «monde » est incarné et représenté dans un village, - géotype le plus symbolique du local par sa petite échelle humaine et sociale, et son organisation sociétale communautaire -, qui est toutefois connecté au monde.

C'est dans cette seconde veine que le chercheur indo-canadien Farokh Afshar a ainsi le premier développé une nouvelle acception du village global (1998), dans la continuité de laquelle je me place ${ }^{16}$. Intéressé par les problématiques de développement en contexte rural, par les conséquences du processus d'urbanisation généralisée, et ainsi conscient de la nécessité de changer le regard porté sur la place des petites localités qui sont également concernées par la mondialisation, au même titre que tout espace, il a donc proposé une définition générique du village global, renvoyant à :

«Tous les petits peuplements reliés, connectés à l'espace mondial par des composants de notre mondialisation courante - l'économie globale, l'écologie, la diversité socio-culturelle, la mobilité humaine, et la technologie incluant les communications. Cela peut s'accompagner d'un sapement de ces peuplements ou d'un renforcement et d'une diffusion de ce qu'au moins certains d'entre eux représentent - i.e. les caractéristiques d'un village telles qu'une échelle plus humaine, un contact plus personnel, une relation symbiotique entre l'environnement naturel et bâti et la gouvernance locale $»^{17}$ (Afshar, 1998, 187).

Certains travaux empiriques se sont intéressés, dans le contexte de la mondialisation, à la mise en valeur spatialement différenciée de villages ou de petites localités, analysée au prisme du village global tel que défini par Afshar (voir Cid-Aguayo, 2008 ; Hill, 2012 ; Shuang, 2012). Toutefois, ces travaux sont relativement peu nombreux. Et aucun d'entre eux n'approche, dans leur cas d'étude, le village global comme prisme d'observation de la vie de migrants internationaux, ni n'interroge directement les enjeux migratoires. Sur ce point, le cadre de lecture du village global, appliqué à Cadaqués, soulève plus d'une question d'intérêt sur l'articulation des échelles et la mise 
en relation des lieux au gré des ancrages et des mobilités. La mondialisation s'incarne dans ce village en même temps que le village se construit en relation avec des univers lointains articulés, dont ceux des migrants: quel est l'apport de la géographie de l'espace local à celle de la mondialisation ? Comment la localité se reconstruit-elle au gré d'apports extérieurs? Et comment les migrants internationaux y participent-ils? Cette relecture du village global vise ainsi plus généralement à comprendre quelles sont les relations entre la migration internationale et les structures évolutives des sociétés, des cultures et des territoires.

\section{Le concept à l'épreuve de l'objet géographique : le village global, géotype}

Mon travail de thèse se différencie d'une analyse monographique traditionnelle. Il invite à tester le concept de village global tel que formalisé par Afshar (1998) à la lumière des transformations connues par Cadaqués. Je compare ces évolutions aux lectures d'autres espaces comme autant de villages globaux dans la littérature scientifique. Je ne cherche donc pas à faire de Cadaqués une exception en soi. Faire du concept de village global un géotype répond à l'exigence de considérer que des configurations spatiales semblables pourraient abriter des réalités sociales fortement distinctes et que, comme le souligne Lévy (2013a, 927) :

«Les objets génériques, modalité qu'empruntent souvent les concepts (ville, pays, etc.), apparaissent comme un entre-deux qui peut laisser la place au singulier sans le réduire à un résidu. Ainsi les «villes mondiales » incluent dans leur définition le fait que leur place dans le Monde résulte en partie d'une "valeur ajoutée " propre à chacune. Il est donc impossible de réduire l'appartenance à la catégorie "ville mondiale " à des critères trop généraux qui pourraient faire manquer le fait décisif : la capacité à disposer d'un monopole ou d'un oligopole sur un ou plusieurs biens d'excellence. »

L'objectif est donc double : d'une part, il s'agit de mettre en exergue un certain nombre de caractéristiques génériques du village global, observables et applicables à d'autres espaces. D'autre part, j'interroge ce qui serait singulier à Cadaqués, comparativement à d'autres localités qualifiées elles aussi de villages globaux. Sur ce point, Lussault (2013, 426) souligne l'intérêt d' "une réflexion conjointe, sur ce qui rapproche deux objets spatiaux (générique), en termes d'organisation, d'agencement, de fonctionnement et, sur ce qui les sépare (spécifique)». Analyser tout espace au prisme d'un objet géographique présente en effet le risque d'estomper les particularités locales, de « surconnoter » cet espace alors qu'il pourrait présenter des réalités différentes de celles d'un autre.

Comment le village global, tel que défini par Afshar (1998), se différencie-t-il alors d'un simple village dans la mondialisation, d'un village connecté ? À partir de quels critères les chercheurs fondent-ils la globalité des villages qu'ils étudient, et qu'ils qualifient alors de "globaux" ? La globalité du village global, qui n'est pas à prendre au sens littéral, est ramenée à certains phénomènes observés; les auteurs de travaux empiriques construisent ainsi distinctement la globalité des villages qu'ils étudient. Ce point révèle d'une part la diversité des phénomènes à l'œuvre d'un contexte villageois à un autre, et d'autre part, la pluralité des acceptions des rapports local-global parmi les chercheurs. 
Pour la sociologue chilienne Beatriz Cid-Aguayo (2010), la globalité d'un village repose sur trois points. Premièrement, cette globalité se fonde sur l'aptitude du village à influencer d'autres localités, à de plus petites échelles spatiales : on parle alors de son rayonnement, de son influence à l'échelle mondiale, à l'échelle du globe, soit, de $s a$ globalité. Deuxièmement, cette globalité s'appuie sur la capacité du pouvoir local à intervenir dans les choix et les décisions qui concernent la mise en valeur et les transformations de son propre espace municipal, concourant au rayonnement du village. Finalement, la globalité du village global repose sur la représentation, la valorisation différenciée (autrement dit, la singularité promue par les acteurs locaux) $\mathrm{du}$ caractère « local » du village. Me plaçant dans cette veine, mon acception du village global renvoie ainsi à «tout village dont les transformations et le rayonnement à de petites échelles spatiales, sinon mondial, traduisent et servent tout ou partie des intérêts du village ».

30 J'ai pu à ce titre isoler deux éléments sur lesquels fonder une globalité de Cadaqués, me permettant ainsi de le qualifier de village global: d'une part au regard des transformations que le village a connu, replacées dans l'histoire récente de la gestion de la valorisation différenciée de ce territoire par le tourisme, vecteur de sa globalité ; d'autre part au prisme de la mobilité des migrants internationaux, dont les trajectoires et les motifs d'ancrage divers à Cadaqués sont révélateurs de la mécanique de fonctionnement propre à ce village global.

\section{Conclusion}

31 Au fil de ce processus itératif qu'est la recherche, le dialogue entre empirie et théorie intervient à différents niveaux de réflexion comme autant d'étapes d'avancement dans la concrétisation du projet de thèse. À l'issue de ce travail réflexif du processus de construction de la thèse, plusieurs constats peuvent être faits. Toute démarche, même inductive, se nourrit d'un contexte théorique de fond. Ce qui peut aller de soi et apparaître évident à la lecture de cet article, ne l'est pas forcément sur les temps courts et longs de la thèse. Présenter de manière chronologique ce processus itératif qui relève souvent du bricolage et qui peut être ponctué de séquences auxquelles toute logique consciente échappe, passe par un exercice de rationalisation auquel doit se prêter le chercheur. Le questionnement intervient tout au long du projet et est rythmé par des allers retours entre empirie et théorie. Il montre dans quelle mesure les changements effectués par tâtonnements, par errements, par hasard ou contrainte même, sont bien une constante dans la réalisation d'un travail de thèse. Ce travail de réflexivité est un processus implicite, sous-jacent, que connaît tout chercheur. Il mériterait d'être systématiquement explicité, de manière à ce qu'il puisse être constructif. Cela permettrait de prendre conscience et de mettre en évidence quels sont les enjeux et les apports particuliers d'un projet de recherche à la lumière de ses failles, de ses incohérences et de ses faiblesses, pour d'autant mieux les dépasser dès lors qu'elles ont été identifiées. 


\section{BIBLIOGRAPHIE}

Afshar, Farokh (1998) Balancing global city with global village, Habitat International, Vol. 22, n4, pp. 375-387.

Appadurai, Arjun (2005) Après le colonialisme. Les conséquences culturelles de la globalisation, Paris, Payot et Rivages, 335 p. (Petite Bibliothèque Payot).

Baby-Collin, Virginie ; Cortes, Geneviève ; Miret, Naïk (2009) Les migrants andins en Espagne, inscriptions spatiales et repérage de filières, Mélanges de la casa de Velázquez, Nouvelle série, Vol. 39, $\mathrm{n}^{\circ} 1$, pp. 115-140.

Bayona-i-Carrasco, Jordi; Gil-Alonso, Fernando (2013) Is Foreign Immigration the Solution to Rural Depopulation? The Case of Catalonia (1996-2009), Sociologia Ruralis, vol. 53, n 1, pp. 26-51.

Benton-Short, Lisa; Price, Marie; Friedman, Samantha (2005) Globalization from Below: The Ranking of Global Immigrant Cities, International Journal of Urban and Regional Research, Vol. 29, nº, pp. 945-959.

Booth, Wayne; Colomb, Gregory; Williams, Joseph (2008) The Craft of Research, Chicago, University of Chicago Press, $3^{\text {ème }}$ édition, 336 p. (Chicago Guides to Writing, Editing, and Publishing).

Carroué, Laurent (2006) Géographie de la mondialisation, Paris, Armand Colin, $2^{2}$ ème édition, 256 p. (U. Géographie).

Castles, Stephen (2010) Understanding Global Migration: A Social Transformation Perspective, Journal of Ethnic and Migration Studies, Vol. 36, n¹0, pp. 1565-1586.

Cid-Aguayo, Beatriz (2008) Global Villages and Rural Cosmopolitanism: Exploring Global Ruralities, Globalizations, Vol. 5, nº 4, pp. 541-554.

Cortes, Geneviève (2000) Partir pour rester. Survie et mutation de sociétés paysannes andines (Bolivie), Paris, IRD Éd., 413 p. (Á Travers Champs).

DeWind, Josh (2007) Preface, in DeSipio, L. ; Garcia y Griego, M. ; Kossoudji ; S. (dir.) Researching Migration: Stories from the Field, New York, Social Science Research Council, pp. 9-12 [réf. du 20 juin 2015] [Disponible sur Internet]

Doceul, Marie-Christine ; Tabarly, Sylviane (2014) Effet de lieu, Géoconfluences [réf. du 20 juin 2015] [Disponible sur Internet]

Dumez, Hervé (2012) Qu'est-ce que l'abduction, et en quoi peut-elle avoir un rapport avec la recherche qualitative ? Le Libellio d'AEGIS, Vol. 8, $\mathrm{n}^{\circ} 3$, pp. 3-9.

Fonseca, Maria Lucinda (2008) New waves of immigration to small towns and rural areas in Portugal, Population, Space and Place, Vol. 14, n6, pp. 525-535.

Fontan, Jean-Marc ; Klein, Juan-Luis ; Tremblay, Diane-Gabrielle (dir.) (1999) Entre la métropolisation et le village global, les scènes territoriales de la reconversion, Sainte-Foy, Presses de l'Université du Québec, 334 p. (Études d'économie politique).

Glick Schiller, Nina; Caglar, Ayse (dir.) (2011) Locating migration rescaling cities and migrants, Ithaca, Cornell University Press, $279 \mathrm{p}$.

Hill, Joseph (2012) The Cosmopolitan Sahara: Building a Global Islamic Village in Mauritania, City \& Society, Vol. 24, n¹, pp. 62-83. 
Hogan, Jackie (2004) Constructing the Global in Two Rural Communities in Australia and Japan, Journal of Sociology, Vol. 40, n¹, pp. 21-40.

Huff, Anne (1999) Writing for Scholarly Publication, ThousandOaks, SAGE Publications Inc, 200 p.

Hugo, Graeme (2008) Immigrant settlement outside of Australia's capital cities, Population, Space and Place, Vol. 14, n6, pp. 553-571.

Hugo, Graeme; Morén-Alegret, Ricard (2008) International migration to non-metropolitan areas of high income countries: editorial introduction, Population, Space and Place, Vol. 14, $\mathrm{n}^{\circ} 6$, pp. 473-477.

Jentsch, Birgit; Simard, Myriam (2009) International Migration and Rural Areas: Cross-National Comparative Perspectives, Burlington, Ashgate, $218 \mathrm{p}$.

Kasimis, Charalambos (2008) Survival and expansion: migrants in Greek rural regions, Population, Space and Place, Vol. 14, nº, pp. 511-524.

Lévy, Jacques (2013a) Singulier/universel, in Lévy, J. ; Lussault, M. (dir.) Dictionnaire de la géographie et de l'espace des sociétés, Paris, Belin, pp. 927-928.

Lévy, Jacques (2013b) Géotype, in Lévy, J. ; Lussault, M. (dir.) Dictionnaire de la géographie et de l'espace des sociétés, Paris, Belin, p. 450.

Lussault, Michel (2013) Générique/spécifique, in Lévy, J. ; Lussault, M. (dir.) Dictionnaire de la géographie et de l'espace des sociétés, Paris, Belin, p. 426.

Mansfield, Becky (2003) Spatializing Globalization: A ‘Geography of Quality’ in the Seafood Industry, Economic Geography, Vol. 79, n¹, pp. 1-16.

Marrow, Helen (2011) New Destination Dreaming: Immigration, Race, and Legal Status in the Rural American South, Stanford, Stanford University Press, 392 p.

McCarthy, James (2008) Rural geography: globalizing the countryside, Progress in Human Geography, Vol. 32, n¹, pp. 129-137.

McLuhan, Marshall (1962) The Gutenberg Galaxy: The Making of Typographic Man, Toronto, University of Toronto Press, 293 p.

Morén-Alegret, Ricard (2008) Ruralphilia and urbophobia versus urbophilia and ruralphobia? Lessons from immigrant integration processes in small towns and rural areas in Spain, Population, Space and Place, Vol. 14, n6, pp. 537-552.

Morén-Alegret, Ricard; Solana, Miguel (2004) Foreign Immigration in Spanish Rural Areas and Small Towns: Current Situation and Perspectives, Finisterra, Vol 39, nº77, pp. 21-38.

Nadler, Robert (2012) Should I stay or should I go? International migrants in the rural town of Zittau (Saxony) and their potential impact on rural development, European Countryside, Vol. 4, $\mathrm{n}^{\circ} 1$, pp. 57-72.

Pierre, Cosette (2009) Publier dans une revue savante, les 10 règles du chercheur convaincant, Québec, Presses de l'Université du Québec, 130 p.

Sassen, Saskia (2001) The Global City: New York, London, Tokyo, Princeton, Princeton University Press, $2^{\text {ème }}$ édition, 472 p. (Princeton Paperbacks).

Shuang, G.A.O. (2012) Commodification of place, consumption of identity: The sociolinguistic construction of a 'global village' in rural China, Journal of Sociolinguistics, Vol. 16, n³, pp. 336-357. 
Simon, Gildas (2008) La planète migratoire dans la mondialisation, Paris, Armand Colin, 256 p. (U. Géographie).

Turabian, Kate (2013) A Manual for Writers of Research Papers, Theses, and Dissertations: Chicago Style for Students and Researchers, Chicago, University Of Chicago Press, $8^{\text {ème }}$ edition, 464 p. (Chicago Guides to Writing, Editing, and Publishing).

Vargas-Silva, Carlos (dir.) (2012) Handbook of Research Methods in Migration, Cheltenham, Edward Elgar Publishing Limited, $608 \mathrm{p}$.

Veltz, Pierre (2014) Mondialisation, villes et territoires : l'économie d'archipel, Paris, PUF, 2 ème édition, 288 p. (Quadrige).

Woods, Michael (2007) Engaging the global countryside: globalization, hybridity and the reconstitution of rural place, Progress in Human Geography, Vol. 31, n²4, pp. 485-507.

\section{NOTES}

1. Cadaqués recense moins de 3000 habitants en l'occurrence $\left(2820\right.$ personnes recensées au $1^{\text {er }}$ janvier 2014, données du Padrón municipal (Padrón Continuo, resultados definitivos), Institut National de Statistiques espagnol, réf. du 20 juin 2015, [Disponible sur Internet]).

2. La vila est définie comme « un peuplement qui, sans avoir été promu au rang de ville, s'était historiquement $\mathrm{vu}$ accorder certains privilèges ou avait un nombre d'habitants tel qu'il permettait à sa municipalité de se distinguer des autres villages ", "Vila » in Gran Enciclopèdia Catalana, (réf. du 20 juin 2015, [Disponible sur Internet]).

3. La localité est entendue ici selon le sens donné par Michel Lussault au terme «local», qui implique pour le chercheur qui s'y intéresse d'adopter « [...] une démarche axiologique qui s'attache à prendre les acteurs au sérieux et à postuler la validité pratique des énoncés qu'ils construisent, à concevoir le local comme qualifiant un ordre de grandeur spatiale relatif à chaque situation interactionnelle observable. [...] Il y aurait donc autant de «niveau local» que de discours de justification de son existence avérés dans une interaction médiatisée par le langage au sein de laquelle l'espace est un enjeu et où le lexique du local constitue une possibilité offerte aux acteurs pour qualifier cet espace en jeu et/ou d'autres espaces de référence » (Lussault, 2013a, 625). Je rejoins aussi la définition de la localité donnée par l'anthropologue indo-américain Arjun Appadurai en tant que « [...] structure de sentiment, propriété de la vie sociale et idéologie d'une communauté identifiée [...]» (2005, 271, traduction libre par l'auteure de l'article).

4. Ce changement se lit dans l'évolution du titre provisoire de ma thèse : «Boliviens de l'Espagne rurale, entre cosmopolitismes ruraux et constructions transnationales, ou la géographie d'un système migratoire international» (2010) à «Habiter et travailler dans un village global : migrations et expériences des Boliviens à Cadaqués » (Catalogne, Espagne), (2013).

5. La mondialisation migratoire est entendue comme «l'extension à l'ensemble de la planète des phénomènes migratoires qui amènent les hommes à changer d'espaces de vie habituels » (Simon, 2008, 6).

6. Traduction libre par l'auteure de l'article.

7. L'effet de lieu est défini comme « la part explicative de l'espace dans les faits économiques et sociaux. Il se pense à toutes les échelles. [...] Les effets de lieu ne doivent pas être limités à une contextualisation des phénomènes, au risque d'une pensée déterministe. Ils sont le fruit d'une multiplicité de processus. » (Doceul, Tabarly, 2014, réf. du 20 juin 2015, [Disponible sur Internet]). 8. Traduction libre de l'auteure de l'article.

9. Les Boliviens composent un tiers environ de la population étrangère totale présente à Cadaqués : sur 834 étrangers, 286 personnes sont de nationalité bolivienne, recensées au $1^{\mathrm{er}}$ 
janvier 2014 : les étrangers représentent ainsi $30 \%$ de la population locale. De leur côté, les Boliviens composent $10 \%$ de la population totale et plus de $30 \%$ de la population étrangère. D’après les données du Padrón municipal (Padrón Continuo, resultados definitivos), Institut National de Statistiques espagnol, (réf. du 20 juin 2015, [Disponible sur Internet]).

10. La population présente à Cadaqués monterait à environ 15000 personnes durant la période estivale, selon le maire Joan Figueras (interview menée en 2012, pour la publication en ligne Alcaldes, (réf. du 20 juin 2015, [Disponible sur Internet]). Les flux touristiques à destination de Cadaqués sont principalement nationaux et en provenance de l'Europe proche (ressortissants français, italiens et anglais majoritairement).

11. La transformation sociale est définie comme « un changement fondamental dans la façon dont la société est organisée et qui va au-delà des processus continus de changement social progressif qui sont toujours à l'œuvre. » (Castles, 2000, 1576, traduction libre de l'auteure de l'article).

12. Traduction libre de l'auteure de l'article.

13. Traduction libre de l'auteure de l'article.

14. Traduction libre de l'auteure de l'article.

15. Un géotype se définit comme une « situation géographique. [...] Un géotype est repéré lorsque l'on traite comme un espace unique un ensemble de configurations spatiales interactives les unes avec les autres. [...] Un géotype peut désigner un espace concret, générique (exemple : la ville) ou bien singulier : c'est alors un géon (exemple : São Paulo) » (Lévy, 2013b, 450).

16. Je m'écarte ainsi d'autres réappropriations du village global, comme par exemple celle de Fontan, Klein et Tremblay (1999) qui s'intéresse plus particulièrement à la mise en valeur des espaces métropolitains, de plus en plus détachés de leur espace national.

17. Traduction libre de l'auteure de l'article.

\section{RÉSUMÉS}

Répondre à une problématique, c'est présenter des résultats de recherche de sorte qu'ils résolvent un problème, géographique ici en l'occurrence, en vue d'apporter une contribution théorique. Cet objectif appelle la mise en œuvre d'une méthodologie rigoureusement pensée (Huff, 1999; Booth, Colomb et Williams, 2008 ; Pierre, 2009; Turabian, 2013). À l'aune de ma participation à diverses rencontres scientifiques portant sur les méthodologies et les méthodes de recherche dans les études migratoires, j'ai pu constater qu'un point récurrent semblait ressortir dans les critiques faites aux participants, suite à l'exposé de leurs analyses. La plupart des chercheurs évoquaient, au gré de leurs expériences, la spécificité de leur terrain et de la construction de leur sujet de recherche, semés d'embuches. Toutefois, nous sommes difficilement à l'aise avec la formulation explicite des répercussions concrètes de ces expériences dans les données collectées, dans la manière de les collecter ou encore dans les analyses que nous en faisons. Ces allers-retours conduisent pourtant à de multiples reformulations dans nos recherches. C'est à ce titre que des travaux dans les études migratoires soulignent l'importance de la réflexivité dans la recherche, pour soumettre au mieux à la critique la production du savoir en sciences humaines et sociales, «la crédibilité des procédés selon lesquels les informations factuelles et les interprétations significatives ont été collectées et produites » (DeWind, 2007, 9). J'aimerais précisément joindre une discussion sur l'importance de l'approche réflexive. Mon sujet de recherche a connu un changement de focus important au gré de mon cheminent intellectuel 
et en réaction aux premiers terrains que j'ai effectués. J'ai à cette occasion recentré mon intérêt sur une seule partie de mes objectifs initiaux. Mon parcours méthodologique a ainsi évolué selon les ajustements nécessaires et possibles à la réalisation de ces objectifs. Je propose de revenir sur ces étapes de construction de ma thèse. Ce processus s'est voulu itératif, entre empirie et théorie.

INDEX

Mots-clés : concept, méthodologie, flux migratoire, milieu rural, conditions sociales, enquête Index géographique : Espagne

\section{AUTEUR}

JOSEPHA MILAZZO

Doctorante en géographie, Aix-Marseille Université (TELEMMe - UMR 7303) ; Universidad Autónoma de Barcelona (Grup de Recerca sobre Migracions)

jjomilazzo@gmail.com 